\title{
THE DUNKL-WILLIAMS INEQUALITY IN AN INNER PRODUCT SPACE
}

\author{
PETER R. MERCER
}

Abstract. We prove a refinement of the well known Dunkl-Williams Inequality in an inner product space.

Mathematics subject classification (2000): Dunkl-Williams inequality. Key words and phrases: 47A30, 26D20.

\section{REFERENCES}

[1] C. F. DunKL, K. S. Williams, A simple norm inequality, Amer. Math. Monthly, 71, (1964), 53-54.

[2] L. MaligrandA, Simple norm inequalities, Amer. Math. Monthly, 113, (2006), 256-260.

[3] J. L. MASSERA, J. J. SCHÄFFER, Linear differential equations and functional analysis I, Ann. of Math., 67, (1958), 517-573.

[4] W. A. KIRK, M. F. SMILEY, Another characterization of inner product spaces, Amer. Math. Monthly, 71, (1964), 890-891. 